\title{
Role of three-nucleon forces and many-body processes in nuclear pairing
}

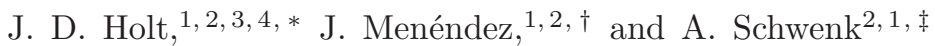 \\ ${ }^{1}$ Institut für Kernphysik, Technische Universität Darmstadt, 64289 Darmstadt, Germany \\ ${ }^{2}$ ExtreMe Matter Institute EMMI, GSI Helmholtzzentrum für Schwerionenforschung GmbH, 64291 Darmstadt, Germany \\ ${ }^{3}$ Department of Physics and Astronomy, University of Tennessee, Knoxville, TN 37996, USA \\ ${ }^{4}$ Physics Division, Oak Ridge National Laboratory, P.O. Box 2008, Oak Ridge, TN 37831, USA
}

\begin{abstract}
We present microscopic valence-shell calculations of pairing gaps in the calcium isotopes, focusing on the role of three-nucleon $(3 \mathrm{~N})$ forces and many-body processes. In most cases, we find a reduction in pairing strength when the leading chiral $3 \mathrm{~N}$ forces are included, compared to results with low-momentum two-nucleon (NN) interactions only. This is in agreement with a recent energy density functional study. At the NN level, calculations that include particle-particle and hole-hole ladder contributions lead to smaller pairing gaps compared with experiment. When particle-hole contributions as well as the normal-ordered one- and two-body parts of $3 \mathrm{~N}$ forces are consistently included to third order, we find reasonable agreement with experimental three-point mass differences. This highlights the important role of $3 \mathrm{~N}$ forces and many-body processes for pairing in nuclei. Finally, we relate pairing gaps to the evolution of nuclear structure in neutron-rich calcium isotopes and study the predictions for the $2^{+}$excitation energies, in particular for ${ }^{54} \mathrm{Ca}$.
\end{abstract}

PACS numbers: 21.10.-k, 21.30.-x, 21.60.Cs, 27.40.+z

\section{INTRODUCTION}

Pairing is a basic component in theoretical descriptions of nuclei 1, 2]. Pairing correlations, experimentally manifested in odd-even mass staggering, can be studied from three-point mass differences, which offer the best compromise between capturing theoretical pairing gaps and excluding contributions from neighboring nuclei. The three-point mass difference for neutrons $\Delta_{n}^{(3)}$ is given by

$\Delta_{n}^{(3)}=\frac{(-1)^{N}}{2}[B(N+1, Z)+B(N-1, Z)-2 B(N, Z)]$,

where $B(N, Z)$ denotes the (negative) ground-state energy of a nucleus with $N$ neutrons and $Z$ protons. This allows comparison between different theoretical approaches and with the experimental $\Delta_{n}^{(3)}$ values obtained directly from mass measurements. Indeed, experimental investigations of exotic nuclei are advancing our understanding of pairing towards the limits of existence [3]. Moreover, since a strong peak in the three-point mass difference indicates a particularly well-bound or magic configuration, it provides information for understanding the evolution of shell structure.

Pairing gaps and global properties of medium-mass to heavy nuclei are best accessed using nuclear energy density functional (EDF) methods [4]. The present EDFs are very successful, but are mainly constrained by fits to nuclei close to stability and therefore can lead to uncontrolled uncertainties in experimentally unexplored regions, especially towards neutron-rich extremes [5]. As a result, recent efforts have focused on the construction and

\footnotetext{
* E-mail: jason.holt@physik.tu-darmstadt.de

$\dagger$ E-mail: javier.menendez@physik.tu-darmstadt.de

‡ E-mail: schwenk@physik.tu-darmstadt.de
}

constraint of EDFs based on microscopic two- (NN) and three-nucleon $(3 \mathrm{~N})$ forces [6 11]. Important steps have also been made in a framework focused on a microscopic treatment of pairing developed from NN and $3 \mathrm{~N}$ interactions, while using existing empirical Skyrme functionals for the remaining part of the EDF 12 15].

Pairing correlations and their influence on the properties of medium-mass nuclei have also been studied within the nuclear shell model [16 20]. To study nuclei towards the limits of existence, we have recently developed microscopic calculations of valence-shell Hamiltonians using many-body perturbation theory (MBPT) based on chiral $\mathrm{NN}$ and $3 \mathrm{~N}$ forces fit only to few-nucleon systems 21 25]. In this approach, it has been shown that $3 \mathrm{~N}$ forces play a key role for the evolution to neutron-rich and proton-rich nuclei [26]: for the location of the neutron dripline in the oxygen isotopes [21, 23], for the ground-state properties and spectra along the proton-rich $Z=8$ and $Z=20$ isotones [25], and for the $N=28$ magic number in calcium [22] and the masses of ${ }^{51,52} \mathrm{Ca}[24]$. The impact of $3 \mathrm{~N}$ forces on neutron-rich nuclei has also been demonstrated in coupled-cluster calculations with phenomenological $3 \mathrm{~N}$ forces 27, 28], in the in-medium similarity renormalization group [29, 30], and using Green's function methods [31].

An important question regarding pairing in nuclei is the contribution of many-body processes, such as medium polarization 32 35]. When Coulomb effects were explicitly included in EDF calculations [13], there was a surprising agreement with experimental data although higher $\mathrm{NN}$ partial waves [36], 3N forces, and the contributions from many-body processes were neglected. When the contribution of $3 \mathrm{~N}$ forces to pairing interactions was recently explored for the first time in EDF calculations [15], this picture, however, changed significantly. It was found that $3 \mathrm{~N}$ forces have a pronounced effect, reducing the pairing gaps systematically by $\sim 30 \%$. 
As a result, the consistent inclusion of many-body processes and whether they can account for the missing pairing strength remain open problems in EDF calculations.

In this work, we address this question in microscopic valence-shell calculations of neutron-rich calcium isotopes that include the contributions from both $3 \mathrm{~N}$ forces and many-body processes consistently. Our work is based on an improved treatment of $3 \mathrm{~N}$ forces [23 25], where the normal-ordered one- and two-body parts of $3 \mathrm{~N}$ forces are included to third order in MBPT (in the first calculations with $3 \mathrm{~N}$ forces 22], their contributions were included only to first order). At the level of particle-particle and hole-hole ladder contributions, which corresponds approximately to the level of EDF calculations, we find that $3 \mathrm{~N}$ forces lead to a reduction in pairing strength comparable to that seen in the EDF results [15], although with an incorrect odd-even staggering of $\Delta_{n}^{(3)}$. This inverted staggering can be related to different mean fields in each approach, which has also been observed in Gorkov-Green's function calculations [37]. When particle-hole contributions are included to third order, reasonable agreement is obtained with experimental three-point mass differences. Finally, we study the $2^{+}$excitation energies based on the same calculations of the even calcium isotopes, and extend the discussion of shell evolution to signatures of shell closures in the pairing gaps. Our improved treatment of $3 \mathrm{~N}$ forces leaves unchanged the prediction of $N=28$ as a magic number [22, 28], but reduces the $2^{+}$excitation energy to $1.7-2.2 \mathrm{MeV}$ in ${ }^{54} \mathrm{Ca}$, which has been recently investigated at RIKEN [38].

\section{MICROSCOPIC VALENCE-SPACE INTERACTIONS AND 3N FORCES}

We calculate the interactions among valence neutrons as in Refs. 23 25], where the theoretical inputs to the valence-shell Hamiltonian, single-particle energies (SPEs) and residual two-body interactions, are calculated microscopically from $\mathrm{NN}$ and $3 \mathrm{~N}$ forces, without adjustments. Our results are based on chiral effective field theory (EFT) interactions, which provide a systematic basis for nuclear forces [39]. At the NN level, we perform a renormalization-group evolution [40, 41] of the $500 \mathrm{MeV} \mathrm{N}{ }^{3} \mathrm{LO}$ potential [42] to a low-momentum cutoff $\Lambda=2.0 \mathrm{fm}^{-1}$ (with smooth regulator 43]) to improve the many-body convergence [41]. The resulting low-momentum interaction $V_{\text {low }} k$ is used to calculate the two-body interactions among valence neutrons to third order in MBPT in a harmonic-oscillator basis of 13 major shells, following the formalism of Refs. [44, 45]. The harmonic-oscillator spacing $\hbar \omega$ is taken to give the same root-mean-square radius as a sphere of uniform density, where in the $p f$ shell we take for $A=42$, $\hbar \omega=41 A^{-1 / 3}=11.48 \mathrm{MeV}$.

At the $3 \mathrm{~N}$ level, we take into account the leading $\mathrm{N}^{2} \mathrm{LO}$ $3 \mathrm{~N}$ forces [46, 47]. This includes the dominant longrange two-pion-exchange parts (due to $\Delta$ and other exci-
TABLE I. Empirical and calculated (MBPT) SPEs in MeV.

\begin{tabular}{c|cc|c}
\hline \hline \multirow{2}{*}{ orbital } & \multicolumn{2}{|c|}{ empirical } & MBPT \\
& GXPF1 [54] & KB3G [53] & $p f g_{9 / 2}$ \\
\hline$f_{7 / 2}$ & -8.62 & -8.60 & -8.05 \\
$p_{3 / 2}$ & -5.68 & -6.60 & -5.86 \\
$p_{1 / 2}$ & -4.14 & -4.60 & -3.22 \\
$f_{5 / 2}$ & -1.38 & -2.10 & -1.33 \\
$g_{9 / 2}$ & $(-1.00)$ & - & -1.23 \\
\hline
\end{tabular}

tations), plus shorter-range one-pion-exchange and contact $3 \mathrm{~N}$ interactions. The short-range $3 \mathrm{~N}$ couplings are fit to the binding energy of ${ }^{3} \mathrm{H}$ and the radius of ${ }^{4} \mathrm{He}$ for $V_{\text {low } k}$ with $\Lambda=\Lambda_{3 \mathrm{~N}}=2.0 \mathrm{fm}^{-1}$ [48]. The fit at lower cutoffs approximately includes the effects of the renormalization-group evolution in the $3 \mathrm{~N}$ sector, up to higher-order $3 \mathrm{~N}$ forces. While at this resolution scale, the impact of $3 \mathrm{~N}$ forces is small in $A=3,4$, they are clearly important in heavier systems. For the two-body interactions among valence neutrons, we include the normalordered two-body part of $3 \mathrm{~N}$ forces consistently to third order in MBPT. This takes into account the interactions of two valence neutrons with a nucleon in the core and gives rise to repulsive interactions between valence neutrons [21, 22]. These contributions are expected to dominate over residual three-body interactions based on phase space arguments for normal Fermi systems 49, as shown recently in the context of the shell model [50]. The normal-ordered two-body approximation has also been benchmarked in ab-initio calculations [51, 52].

The SPEs in ${ }^{41} \mathrm{Ca}$ are obtained by solving the Dyson equation, including one-body contributions to third order in MBPT in the same space as the two-body interactions. We include the normal-ordered one-body part of $3 \mathrm{~N}$ forces, which corresponds to the interactions of one valence neutron with two nucleons in the core. These $3 \mathrm{~N}$ contributions range from $1-6 \mathrm{MeV}$, depending on the orbital, approximately an order of magnitude larger than two-body effects, which is consistent with the normalordering hierarchy [49, 51, 52]. The convergence in the one-body sector is slower than the two-body case, but the results show convergence in 13 major shells for the NN part of nuclear forces, and in 5 major shells for the $3 \mathrm{~N}$ part. At each self-consistency iteration when solving the Dyson equation, the unperturbed harmonicoscillator spectrum is updated such that the energy of the degenerate valence-space orbitals is set to the centroid, $\sum_{j}(2 j+1) \varepsilon_{j} / \sum_{j}(2 j+1)$, of the calculated SPEs of the previous iteration. The calculated SPEs are given in Table [1. They are comparable to those of the phenomenological KB3G [53] and GXPF1 [54] interactions for the $p f$-shell orbitals 22].

We have previously shown that, in valence-shell calculations based on nuclear forces, the inclusion of orbitals on top of the standard one major harmonic- 


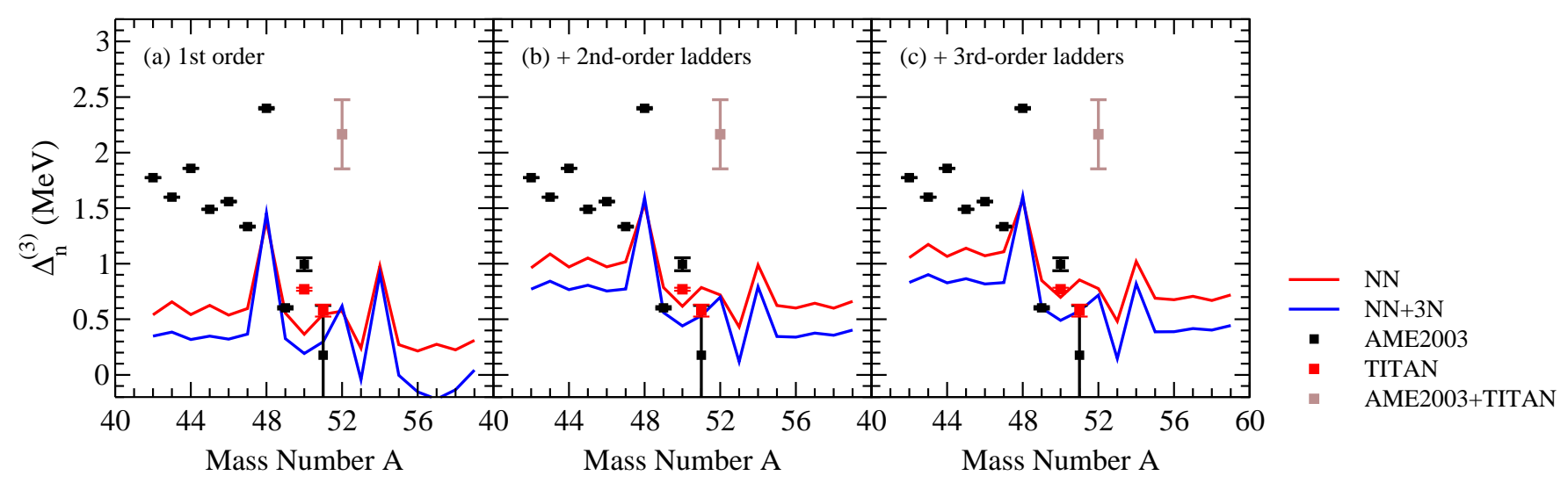

FIG. 1. (Color online) Three-point mass differences $\Delta_{n}^{(3)}$ from ${ }^{40} \mathrm{Ca}$ to ${ }^{60} \mathrm{Ca}$ calculated in the $p f$-shell to successive orders of particle-particle and hole-hole ladders, with and without the leading chiral $3 \mathrm{~N}$ forces, and in comparison with experiment. Panel (a) shows the first-order pairing gaps, while panels (b) and (c) include the effects of adding second- and third-order ladder contributions, respectively. All calculations use empirical SPEs in ${ }^{41} \mathrm{Ca}$ (from GXPF1 [54]). Experimental data are from the 2003 Atomic Mass Evaluation (AME2003) [67] and including recent precision mass measurements for ${ }^{51,52}$ Ca from TITAN [24].

oscillator shell becomes important for neutron-rich isotopes 22, 23]. This also is in line with our calculational framework, which treats the valence orbitals exactly by diagonalization, but includes the contributions from outside the valence space perturbatively. Therefore, extended valence spaces treat nonperturbatively as many orbitals as possible in the diagonalization. To probe the effects on pairing of the correlations involving extendedspace orbitals, we have performed calculations in both the $0 f_{7 / 2}, 1 p_{3 / 2}, 0 f_{5 / 2}, 1 p_{1 / 2}$ valence space $(p f$-shell $)$ as well as the expanded space including the $0 g_{9 / 2}$ orbital $\left(p f g_{9 / 2}\right.$ valence space). Both cases start from a ${ }^{40} \mathrm{Ca}$ core. We take two approaches with respect to SPEs: in all $p f$-shell calculations we use the empirical SPEs of Ref. [54], while for the $p f g_{9 / 2}$ valence space, we either use these empirical values (with the $g_{9 / 2}$ SPE set to $-1.0 \mathrm{MeV}$ ), or the SPEs calculated consistently in MBPT. Regarding the $p f g_{9 / 2}$ calculations, in Table $\square$ we observe that the $g_{9 / 2}$ orbital is obtained close to the $p f$ shell, which can lead to substantial contributions from this orbital.

Since the $p f g_{9 / 2}$ valence space comprises orbitals beyond one major harmonic-oscillator shell, the center-ofmass $(\mathrm{cm})$ motion of the valence nucleons is not guaranteed to factorize in general. As in Refs. [55, 56], we explore possible $\mathrm{cm}$ admixture in our calculations by adding a cm Hamiltonian, $\beta H_{\mathrm{cm}}$, with $\beta=0.5$, to the valenceshell Hamiltonian. This results in only modest changes of $\lesssim 300 \mathrm{keV}$ in the pairing gaps. These changes can be understood because the non-zero cm two-body matrix elements are also relevant matrix elements of the MBPT calculation, making a separation between these two effects difficult. Similarly, we find nonzero $\left\langle H_{c m}\right\rangle$ values, which point to possible $\mathrm{cm}$ admixture and/or nonneglible occupancies of the $g_{9 / 2}$ orbital. However, as discussed in Ref. [57] and shown in the context of coupled-cluster calculations [58], $\beta$-dependence and $\left\langle H_{c m}\right\rangle \neq 0$ do not necessarily imply $\mathrm{cm}$ contamination.

Since it is important to understand this issue, work is in progress in several directions in both the $p f g_{9 / 2}$ and $s d f_{7 / 2} p_{3 / 2}$ [23] valence spaces. First, we perform a nonperturbative Okubo-Lee-Suzuki-Okamoto transformation 5962 to project the extended valence-space interaction into the standard major harmonic-oscillator shell, which is free of $\mathrm{cm}$ spurious states. This will nonperturbatively include the contributions from the extended orbitals. We will also adapt the singular-value decomposition used in Ref. [58] to test $\mathrm{cm}$ factorization in the extended-space calculations. Finally, we will apply the in-medium similarity renormalization group (IMSRG) 63, 64] to develop extended valence spaces, where the cross-shell matrix elements can be suppressed under the evolution to the valence-shell Hamiltonian. This will ensure $\left\langle H_{\mathrm{cm}}\right\rangle \approx 0$ and provide a nonperturbative benchmark for MBPT calculations.

With the developed valence-shell interactions based on chiral $\mathrm{NN}$ and $3 \mathrm{~N}$ forces, we perform exact diagonalizations in the valence space to obtain the ground states and, for the even calcium isotopes, the first excited $2^{+}$ states. The shell-model codes ANTOINE [17, 65] and NATHAN [17] have been used throughout this work.

\section{RESULTS}

\section{A. Effects of $3 \mathrm{~N}$ forces and many-body processes}

The EDF pairing calculations of Ref. [15] use the same chiral $\mathrm{NN}$ and $3 \mathrm{~N}$ forces of Sect. III where $3 \mathrm{~N}$ forces are included as density-dependent two-body interactions from Ref. 66], and restricts the contributions to pairing to the dominant ${ }^{1} \mathrm{~S}_{0}$ channel. Pairing in the 


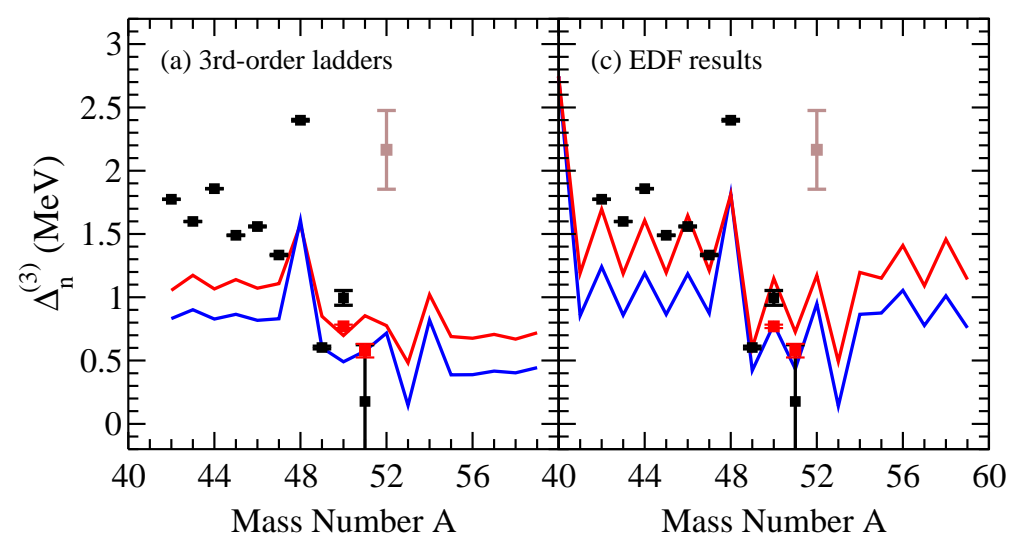

FIG. 2. (Color online) Three-point mass differences $\Delta_{n}^{(3)}$ from ${ }^{40} \mathrm{Ca}$ to ${ }^{60} \mathrm{Ca}$ calculated to third-order ladders in $\mathrm{MBPT}$ with empirical SPEs, panel (a), compared with the EDF results of Ref. [15], panel (b). Results with and without the leading chiral $3 \mathrm{~N}$ forces are shown following the legend of Fig. 1] and in comparison with experiment [24, 67].

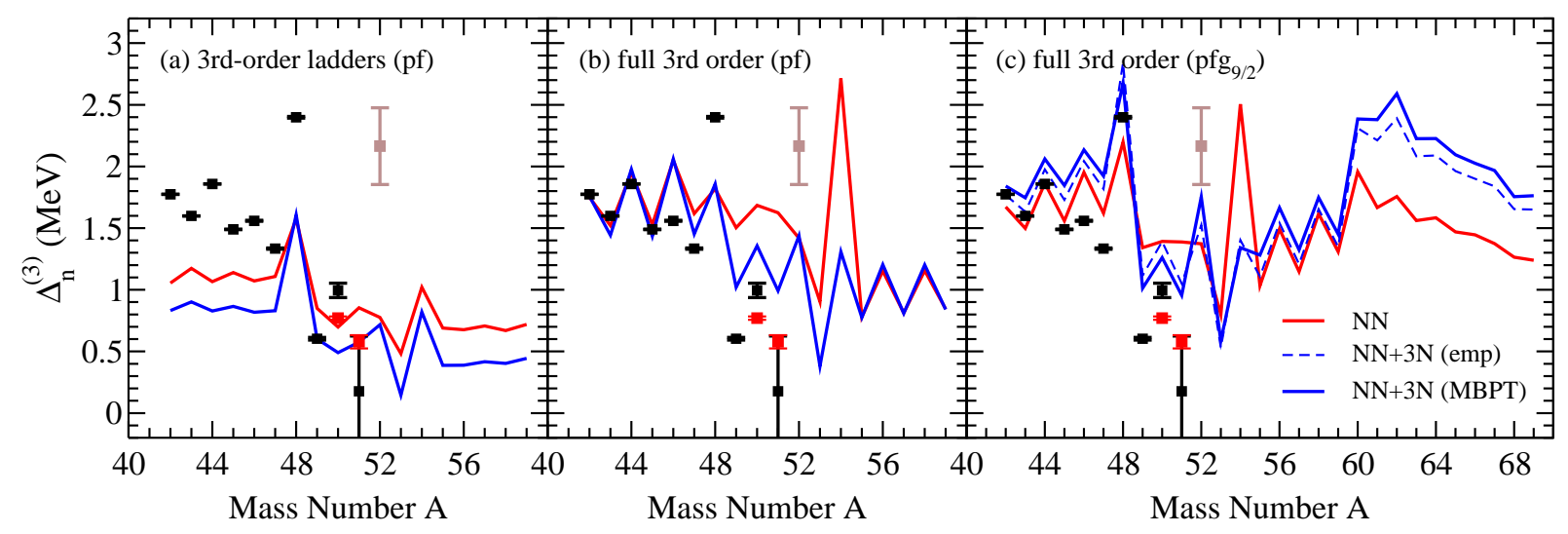

FIG. 3. (Color online) Three-point mass differences $\Delta_{n}^{(3)}$ in the calcium isotopes calculated to third order in MBPT with and without the leading chiral 3N forces, and in comparison with experiment [24, 67]. The legend is as in Fig. 11 Panel (a) shows the results of the third-order ladder contributions. Panels (b) and (c) include all MBPT diagrams to third order in the $p f$-shell and the extended $p f g_{9 / 2}$ valence space, respectively. The results in the $p f$-shell are with empirical SPEs. For the $p f g_{9 / 2}$ space, we show pairing gaps for both the MBPT and empirical SPEs.

EDF approach is realized by solving the Hartree-FockBogoliubov equations, which in the pairing channel approximately is equivalent to summing ladder diagrams. Although the comparison to diagonalization in the valence space is not one-to-one, it is still instructive to first compare EDF results to valence-shell calculations including particle-particle and hole-hole ladder diagrams only.

Figure 11 shows the three-point mass differences $\Delta_{n}^{(3)}$ from ${ }^{40} \mathrm{Ca}$ to ${ }^{60} \mathrm{Ca}$ calculated in the $p f$-shell to successive orders of particle-particle and hole-hole ladders. At both the $\mathrm{NN}$ and $\mathrm{NN}+3 \mathrm{~N}$ level, increasing the order to which ladders are included also increases the pairing gaps, systematically improving the agreement with experimental data taken from the 2003 Atomic Mass Evaluation (AME2003) 67] (black points) and improved high-precision mass measurements for ${ }^{51,52} \mathrm{Ca}$ from TITAN [24] (red points), which for ${ }^{52} \mathrm{Ca}$ deviate from the inaccurate AME2003 value by $1.74 \mathrm{MeV}$. Moreover, the results converge rapidly: from first to second order, there is a significant increase in $\Delta_{n}^{(3)}$ of $\sim 0.4 \mathrm{MeV}$; from second to third order, the change is only $\sim 0.1 \mathrm{MeV}$.

Pairing gaps calculated at this level are clearly deficient with respect to experiment. In addition to being below the experimental pairing strength, the odd-even staggering of $\Delta_{n}^{(3)}$ is inverted compared to experiment $\left(\Delta_{n}^{(3)}\right.$ is stronger for odd masses than for even ones). This inverted staggering is a sign that the mean-field part at this level is too attractive, resulting in a lack of saturation and an incorrect symmetry energy, similar to the calculations discussed in Ref. [37]. The correct odd-even staggering of $\Delta_{n}^{(3)}$ seen in the EDF results of Ref. 15] (see the right panel of Fig. 2) is already built into the Skyrme functional used in Ref. [15].

Taking into account $3 \mathrm{~N}$ force contributions at the lad- 


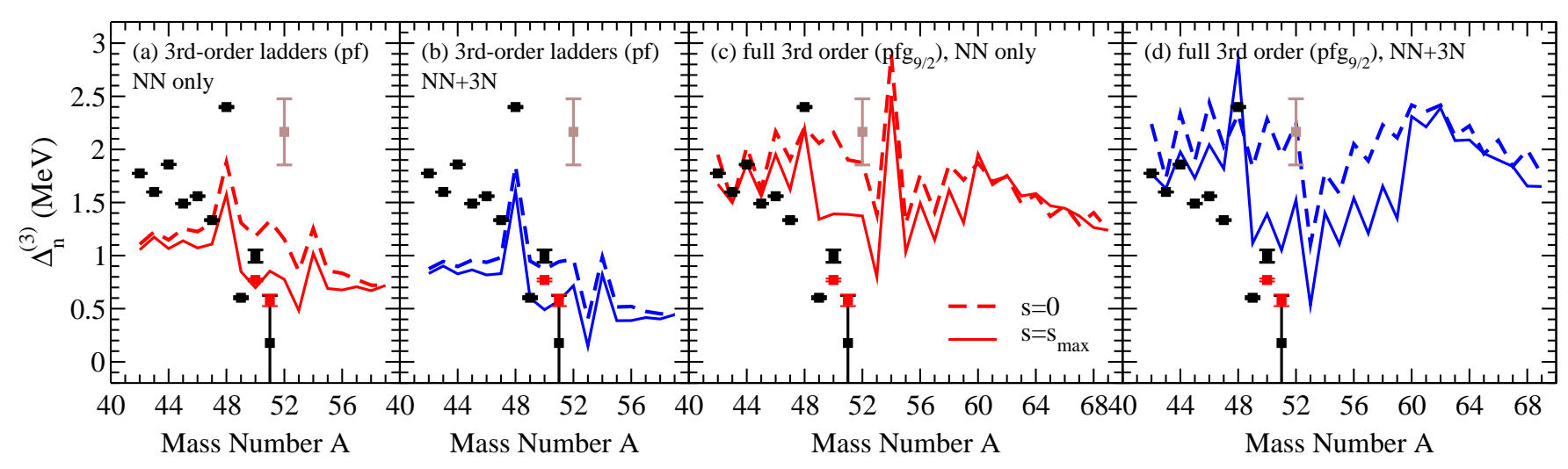

FIG. 4. (Color online) Three-point mass differences $\Delta_{n}^{(3)}$ in the calcium isotopes calculated to third order in MBPT with and without the leading chiral $3 \mathrm{~N}$ forces, and in comparison with experiment 24, 67]. The full calculations (solid lines) are compared to the case where all valence neutrons are coupled to $J=0$ pairs (seniority $s=0$, given by the dashed lines). Panel (a) shows results based on NN forces only in the $p f$-shell, while panel (b) includes NN+3N interactions. Similarly, panels (c) and (d) show results in the extended $p f g_{9 / 2}$ valence space including $\mathrm{NN}$ and $\mathrm{NN}+3 \mathrm{~N}$ interactions, respectively. All calculations use empirical SPEs in ${ }^{41} \mathrm{Ca}$ (from GXPF1 [54]).

ders level, we find in Fig. 1 that the repulsive effect of chiral $3 \mathrm{~N}$ forces leads to a systematic suppression of $\Delta_{n}^{(3)}$. Ranging from $0.2-0.5 \mathrm{MeV}$, this is similar to the decrease in pairing strength observed in the EDF study of Ref. [15], as can be seen in Fig. 2, Note that the incorrect odd-even staggering of $\Delta_{n}^{(3)}$ persists in the $\mathrm{NN}+3 \mathrm{~N}$ case, but the repulsive $3 \mathrm{~N}$ forces make it less pronounced. Moreover, as shown in Fig. 3. the impact of $3 \mathrm{~N}$ forces on pairing gaps varies more along the isotopic chain, when all third-order many-body processes are included.

When particle-hole contributions are included in a full third-order calculation, we find in Fig. 3 a clear improvement compared to including only ladder diagrams. In the $p f$-shell, the three-point mass differences are increased, leading to reasonable agreement with experimental data. This clearly demonstrates the importance of particle-hole many-body processes, such as core-polarization, on pairing in nuclei. Our results show that they can provide the missing pairing strength required to reproduce experiment on top of the direct $\mathrm{NN}+3 \mathrm{~N}$ interactions. Analogously, the systematic differences between theoretical and experimental pairing gaps found in the EDF approach of Ref. [15] may be attributed to these effects.

From Fig. 3 (b) and (c) we see that $3 \mathrm{~N}$ forces have a limited effect in the full calculation from ${ }^{42-47} \mathrm{Ca}$ and ${ }^{55-59} \mathrm{Ca}$. In the first region, experimental threepoint mass differences are already well described with NN forces only. More noticeable differences occur from ${ }^{48-54} \mathrm{Ca}$, where the addition of $3 \mathrm{~N}$ forces significantly modifies the structure of $\Delta_{n}^{(3)}$. In particular the rather flat trend in pairing strength from ${ }^{48-52} \mathrm{Ca}$ given by NN forces evolves to a structure with peaks at the even isotopes, reproducing qualitatively the experimental oddeven staggering in this region 24]. Extending the calculation from the $p f$-shell to the $p f g_{9 / 2}$ valence space provides further improvement when compared to experi- ment, in particular with respect to the pronounced peaks at ${ }^{48} \mathrm{Ca}$ and ${ }^{52} \mathrm{Ca}$ and the corresponding shell closures. On the other hand, this overestimates $\Delta_{n}^{(3)}$ at ${ }^{45-47} \mathrm{Ca}$, but most likely this is due to the influence of the somewhat overpredicted shell closure at ${ }^{48} \mathrm{Ca}$ rather than a deficiency in the pairing channel. We will discuss shell structure further in Sect. IIIC.

\section{B. Seniority truncations}

We can further study the physics of the pairing gaps by considering a restricted calculation, in which the full third-order wavefunctions are constrained to have all valence neutrons forming $J=0$ pairs (seniority $s=0$ ).

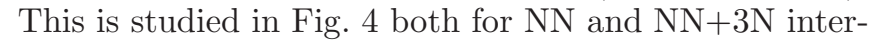
actions in the $p f$-shell and the $p f g_{9 / 2}$ valence space. In most cases, at the $s=0$ level there is already a reasonable description of the three-point mass differences, indicating that pairing gaps are, as expected, largely described by $J=0$ interactions. This is also reflected when using phenomenological interactions [53, 54], where the difference of the $s=0$ to the full calculation is $\lesssim 100 \mathrm{keV}$. However, for mid-shell isotopes around ${ }^{50} \mathrm{Ca}$ there is a clear difference for the interactions studied here, especially when including $3 \mathrm{~N}$ forces in the $p f g_{9 / 2}$ valence space. This gives us an indication how the $g_{9 / 2}$ orbit is affecting physics in this region, as we find that the main difference in going from $s=0$ to $s=s_{\max }$ is in the occupancy of this orbital. At the level $s=4$, where all but 4 particles are constrained to form $J=0$ pairs, the results are nearly indistinguishable from those of the full calculation. 


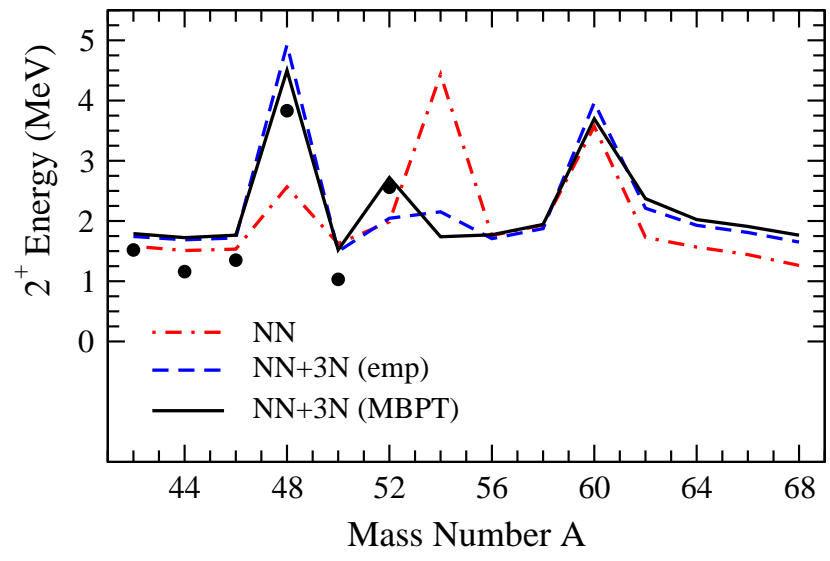

FIG. 5. (Color online) First $2^{+}$excitation energies in the even calcium isotopes with and without $3 \mathrm{~N}$ forces compared with experiment. The energies are calculated to ${ }^{68} \mathrm{Ca}$ in the extended $p f g_{9 / 2}$ valence space, using both empirical (dotdashed and dashed lines) and microscopic MBPT SPEs (solid line). Experimental energies are taken from Ref. 68].

\section{Pairing gaps and shell closures}

A well-known signature of a closed-shell nucleus is a relative peak in the three-point mass difference. Therefore, this work extends the discussion of the evolution of shell structure in the neutron-rich calcium isotopes [22, 28]. We study the $2^{+}$excitation energies of the even calcium isotopes based on the same calculations as for the three-point mass differences presented here and in Ref. [24], where 3N force contributions are included consistently to third order in MBPT. The resulting $2^{+}$ excitation energies are presented in Fig. 5 .

From Fig. 3 (b) we observe that the pairing gaps calculated in the $p f$-shell largely follow the results of Ref. [22], where no apparent closure is seen in ${ }^{48} \mathrm{Ca}$ with $\mathrm{NN}$ forces and limited enhancement when $3 \mathrm{~N}$ forces are included. Furthermore, with the addition of $3 \mathrm{~N}$ forces, a very modest peak emerges at ${ }^{52} \mathrm{Ca}$, and an initially strong peak at ${ }^{54} \mathrm{Ca}$ becomes strongly suppressed. It is very interesting that at ${ }^{52} \mathrm{Ca}, \Delta_{n}^{(3)}$ obtained from a combination of masses from the recent precision measurements of ${ }^{51,52} \mathrm{Ca}$ from TITAN [24] and the AME2003 extrapolation [67] displays a signature of a shell closure at ${ }^{52} \mathrm{Ca}$, consistent with the high experimental $2^{+}$energy. However, direct mass measurements beyond ${ }^{52} \mathrm{Ca}$ are critical.

In the $p f g_{9 / 2}$ extended space calculations of Fig. 3(c), we find a clear enhancement at ${ }^{48} \mathrm{Ca}$ with $3 \mathrm{~N}$ forces, suggesting that correlations involving the $g_{9 / 2}$ orbit are important in predicting this shell closure. In Fig. 5, we similarly find a high $2^{+}$excitation energy, which is somewhat overpredicted. The best agreement with experiment in Fig. 5 is found for the NN+3N case with MBPT SPEs, where all inputs to the valence-shell Hamiltonian are calculated based on chiral NN and $3 \mathrm{~N}$ forces without adjustments. In ${ }^{54} \mathrm{Ca}$, Figs. 3 and 5 show that $3 \mathrm{~N}$ forces lead to a quenching of the pairing gap strength and a lowering of the $2^{+}$energy (to $1.7-2.2 \mathrm{MeV}$ ), regardless of the valence space or which SPEs are used. This is in contrast to the calculations of Ref. [22], based on first-order $3 \mathrm{~N}$ force contributions. This shows that the structure of $N=34$ is sensitive to theoretical details of the calculation, making this a very good test case, as discussed in Ref. [28]. Further investigations are necessary to explore the theoretical uncertainties from the truncation of $3 \mathrm{~N}$ forces and to the valence space.

As we approach ${ }^{70} \mathrm{Ca}$, we see no further signatures of shell closures in the pairing gaps, except for the interesting case of ${ }^{60} \mathrm{Ca}$, which corresponds to a potential $N=40$ magic number. In this case, regardless of whether $3 \mathrm{~N}$ forces are taken into account, there is a significant peak in $\Delta_{n}^{(3)}$. This is supported by the results of Fig. 0 , where the calculated $2^{+}$energies are approximately twice the $2^{+}$energies in ${ }^{58} \mathrm{Ca}$ and ${ }^{62} \mathrm{Ca}$. This result should, however, be taken with care, because of the theoretical uncertainties from the truncation of the valence space and from neglected continuum effects, which both are more severe for the most neutron-rich calcium isotopes. Recent coupled-cluster calculations indicate that due to the continuum, the higher-lying $1 d_{5 / 2}$ and $2 s_{1 / 2}$ orbitals, not considered in this calculation, are lowered significantly at ${ }^{60} \mathrm{Ca}$, both appearing below the $g_{9 / 2}$ orbital [28].

Finally, it is worth emphasizing that in Fig. 2] while the ${ }^{40} \mathrm{Ca}$ and to a lesser extent the ${ }^{48} \mathrm{Ca}$ shell closures are evident in the EDF results, there is little indication of shell structure throughout the rest of the isotopic chain. Indeed, there is also no clear peak in $\Delta_{n}^{(3)}$ for either ${ }^{52} \mathrm{Ca}$ or ${ }^{54} \mathrm{Ca}$ in the ladder results of Fig. 2. While the addition of $3 \mathrm{~N}$ forces does strengthen the peak signature in ${ }^{48} \mathrm{Ca}$, the presence of the peak with $\mathrm{NN}$ forces is mainly due to the very large spacing of the $f_{7 / 2}$ and $p_{3 / 2}$ orbitals $(\sim 4 \mathrm{MeV})$ in the EDF SPEs [69].

\section{SUMMARY}

We have studied the role of $3 \mathrm{~N}$ forces and many-body processes in a microscopic description of nuclear pairing in the calcium isotopes. At the level of particle-particle and hole-hole ladder contributions, the repulsion from $3 \mathrm{~N}$ forces decreases the pairing gaps, in line with the EDF calculations of Ref. [15]. Taking into account many-body processes to third order in MBPT, we have found reasonable agreement with experimental three-point mass differences. This shows the important role of attractive particle-hole contributions to pairing in nuclei [32 35] in our first calculation based on chiral $\mathrm{NN}$ and $3 \mathrm{~N}$ forces. Finally, we have studied the $2^{+}$excitation energies of the even calcium isotopes in the same framework. This improves the first calculations with $3 \mathrm{~N}$ forces [22], by including $3 \mathrm{~N}$ force contributions consistently to third order in MBPT as in Ref. [24]. This improved treatment leaves unchanged the prediction of $N=28$ as a magic number [22, 28], but reduces the $2^{+}$excitation energy to 
$1.7-2.2 \mathrm{MeV}$ in ${ }^{54} \mathrm{Ca}$, which has been recently investigated at RIKEN [38].

Although this study focused on the calcium isotopes, extensions to heavier isotopic chains such as nickel and tin are possible. They will serve as a test of the presented valence-shell calculations in heavy nuclei, while providing a further link between the shell model and EDF methods based on nuclear forces.

\section{ACKNOWLEDGMENTS}

We thank S. K. Bogner, T. Duguet, T. Lesinski, and V. Somà for useful discussions. This work was supported by the BMBF under Contract No. 06DA70471, the DFG through Grant SFB 634, the Helmholtz Association through the Helmholtz Alliance Program, contract HA216/EMMI "Extremes of Density and Temperature: Cosmic Matter in the Laboratory", and the US DOE Grants DE-FC02-07ER41457 (UNEDF SciDAC Collaboration) and DE-FG02-96ER40963. Computations were performed with an allocation of advanced computing resources on Kraken at the National Institute for Computational Sciences and at the Jülich Supercomputing Center.
[1] A. Bohr, B. R. Mottelson, and D. Pines, Phys. Rev. 110, 936 (1958).

[2] A. Bohr and B. R. Mottelson, Nuclear Structure, Vol. 1 (W. A. Benjamin, New York, 1969).

[3] J. Dobaczewski and W. Nazarewicz, Prog. Theor. Phys. Suppl. 146, 70 (2003).

[4] M. Bender, P.-H. Heenen, and P.-G. Reinhard, Rev. Mod. Phys. 75, 121 (2003).

[5] G. F. Bertsch, C. A. Bertulani, W. Nazarewicz, N. Schunck, and M. V. Stoitsov, Phys. Rev. C 79, 034306 (2009).

[6] S. K. Bogner, R. J. Furnstahl, and L. Platter, Eur. Phys. J. A 39, 219 (2009).

[7] J. E. Drut, R. J. Furnstahl, and L. Platter, Prog. Part. Nucl. Phys. 64, 120 (2010).

[8] B. Gebremariam, T. Duguet, and S. K. Bogner, Phys. Rev. C 82, 014305 (2010).

[9] M. Stoitsov, M. Kortelainen, S. K. Bogner, T. Duguet, R. J. Furnstahl, B. Gebremariam, and N. Schunck, Phys. Rev. C 82, 054307 (2010).

[10] J. E. Drut and L. Platter, Phys. Rev. C 84, 014318 (2011).

[11] S. K. Bogner, R. J. Furnstahl, H. Hergert, M. Kortelainen, P. Maris, M. Stoitsov, and J. P. Vary, Phys. Rev. C 84, 044306 (2011).

[12] T. Duguet and T. Lesinski, Eur. Phys. J. ST 156, 207 (2008).

[13] T. Lesinski, T. Duguet, K. Bennaceur, and J. Meyer, Eur. Phys. J. A 40, 121 (2009).

[14] K. Hebeler, T. Duguet, T. Lesinski, and A. Schwenk, Phys. Rev. C 80, 044321 (2009).

[15] T. Lesinski, K. Hebeler, T. Duguet, and A. Schwenk, J. Phys. G 39, 015108 (2012).

[16] D. J. Dean and M. Hjorth-Jensen, Rev. Mod. Phys. 75, 607 (2003).

[17] E. Caurier, G. Martínez-Pinedo, F. Nowacki, A. Poves, and A. P. Zuker, Rev. Mod. Phys. 77, 427 (2005).

[18] E. Caurier, J. Menéndez, F. Nowacki, and A. Poves, Phys. Rev. Lett. 100, 052503 (2008).

[19] K. Kaneko, J. Zhang, and Y. Sun, Phys. Lett. B 671, 42 (2009).

[20] B. A. Brown, in Fifty Years of Nuclear BCS - Pairing in Finite Systems, Eds. R. A. Broglia and V. Zelevinsky
(World Scientific, 2013)

[21] T. Otsuka, T. Suzuki, J. D. Holt, A. Schwenk, and Y. Akaishi, Phys. Rev. Lett. 105, 032501 (2010).

[22] J. D. Holt, T. Otsuka, A. Schwenk, and T. Suzuki, J. Phys. G 39, 085111 (2012).

[23] J. D. Holt, J. Menéndez, and A. Schwenk, Eur. Phys. J. A 49, 39 (2013).

[24] A. T. Gallant et al., Phys. Rev. Lett. 109, 032506 (2012).

[25] J. D. Holt, J. Menéndez, and A. Schwenk, Phys. Rev. Lett. 110, 022502 (2013).

[26] H.-W. Hammer, A. Nogga, and A. Schwenk, Rev. Mod. Phys. 85, 197 (2013).

[27] G. Hagen, M. Hjorth-Jensen, G. R. Jansen, R. Machleidt, and T. Papenbrock, Phys. Rev. Lett. 108, 242501 (2012).

[28] G. Hagen, M. Hjorth-Jensen, G. R. Jansen, R. Machleidt and T. Papenbrock, Phys. Rev. Lett. 109, 032502 (2012).

[29] H. Hergert, S. K. Bogner, S. Binder, A. Calci, J. Langhammer, R. Roth, and A. Schwenk, Phys. Rev. C 87, 034307 (2013).

[30] H. Hergert, S. Binder, A. Calci, J. Langhammer, and R. Roth, arXiv:1302.7294.

[31] A. Cipollone, C. Barbieri, and P. Navrátil, arXiv:1303.4900

[32] J. Terasaki, F. Barranco, R. A. Broglia, E. Vigezzi, and P. F. Bortignon, Nucl. Phys. A 697, 127 (2002).

[33] F. Barranco, R. A. Broglia, G. Colò, E. Vigezzi, and P. F. Bortignon, Eur. Phys. J. A 21, 57 (2004).

[34] G. Gori, F. Ramponi, F. Barranco, P. F. Bortignon, R. A. Broglia, G. Colò, and E. Vigezzi, Phys. Rev. C 72 , 011302 (2005).

[35] A. Pastore, F. Barranco, R. A. Broglia, and E. Vigezzi, Phys. Rev. C 78, 024315 (2008).

[36] S. Baroni, A. O. Macchiavelli, and A. Schwenk, Phys. Rev. C 81, 064308 (2010).

[37] T. Duguet, in Fifty Years of Nuclear BCS - Pairing in Finite Systems, Eds. R. A. Broglia and V. Zelevinsky (World Scientific, 2013), arXiv:1204.2737, and V. Somà, private communication.

[38] D. Steppenbeck et al., in preparation.

[39] E. Epelbaum, H.-W. Hammer, and U.-G. Meißner, Rev. Mod. Phys. 81, 1773 (2009).

[40] S. K. Bogner, T. T. S. Kuo, and A. Schwenk, Phys. Rept. 386, 1 (2003). 
[41] S. K. Bogner, R. J. Furnstahl, and A. Schwenk, Prog. Part. Nucl. Phys. 65, 94 (2010).

[42] D. R. Entem and R. Machleidt, Phys. Rev. C 68, 041001(R) (2003).

[43] S. K. Bogner, R. J. Furnstahl, S. Ramanan, and A. Schwenk, Nucl. Phys. A 784, 79 (2007).

[44] T. T. S. Kuo and E. Osnes, Springer Lecture Notes of Physics, Vol. 364, p. 1 (1990).

[45] M. Hjorth-Jensen, T. T. S. Kuo, and E. Osnes, Phys. Rept. 261, 125 (1995).

[46] U. van Kolck, Phys. Rev. C 49, 2932 (1994).

[47] E. Epelbaum, A. Nogga, W. Glöckle, H. Kamada, U.-G. Meißner, and H. Witała, Phys. Rev. C 66, 064001 (2002).

[48] S. K. Bogner, R. J. Furnstahl, A. Nogga, and A. Schwenk, arXiv:0903.3366 K. Hebeler, S. K. Bogner, R. J. Furnstahl, A. Nogga, and A. Schwenk, Phys. Rev. C 83 031301(R) (2010).

[49] B. Friman and A. Schwenk, in From Nuclei to Stars: Festschrift in Honor of Gerald E. Brown, Ed. S. Lee (World Scientific, 2011) arXiv:1101.4858.

[50] C. Caesar, J. Simonis et al., arXiv:1209.0156.

[51] G. Hagen, T. Papenbrock, D. J. Dean, A. Schwenk, A. Nogga, M. Włoch, and P. Piecuch, Phys. Rev. C 76, 034302 (2007).

[52] R. Roth, S. Binder, K. Vobig, A. Calci, J. Langhammer, and P. Navrátil, Phys. Rev. Lett. 109, 052501 (2012).

[53] A. Poves, J. Sánchez-Solano, E. Caurier, and F. Nowacki, Nucl. Phys. A 694, 157 (2001).

[54] M. Honma, T. Otsuka, B. A. Brown, and T. Mizusaki,
Phys. Rev. C 69, 034335 (2004).

[55] D. J. Dean, M. T. Ressell, M. Hjorth-Jensen, S. E. Koonin, K. Langanke, and A. P. Zuker, Phys. Rev. C 59, 2474 (1999).

[56] E. Caurier, J. Menéndez, F. Nowacki, and A. Poves, Phys. Rev. C 75, 054317 (2007).

[57] J. B. McGrory and B. H. Wildenthal, Phys. Lett. B 60, 5 (1975).

[58] G. Hagen, T. Papenbrock, and D. J. Dean, Phys. Rev. Lett. 103, 062503 (2009).

[59] S. Okubo, Prog. Theor. Phys. 12, 603 (1954).

[60] S. Y. Lee and K. Suzuki, Phys. Lett. B 91, 173 (1980).

[61] K. Suzuki and S. Y. Lee, Prog. Theor. Phys. 64, 2091 (1980).

[62] K. Suzuki and R. Okamoto, Prog. Theor. Phys. 70, 439 (1983)

[63] K. Tsukiyama, S. K. Bogner, and A. Schwenk, Phys. Rev. Lett. 106, 222502 (2011).

[64] K. Tsukiyama, S. K. Bogner, and A. Schwenk, Phys. Rev. C 85, 061304(R) (2012).

[65] E. Caurier and F. Nowacki, Acta Phys. Pol. 30, 705 (1999).

[66] K. Hebeler and A. Schwenk, Phys. Rev. C 82, 014314 (2010).

[67] G. Audi, A. H. Wapstra, and C. Thibault, Nucl. Phys. A 729, 337 (2003).

[68] http://www.nndc.bnl.gov/ensdf/

[69] T. Lesinski, private communication. 